Janis Dinter ${ }^{*}$, Vanessa Färber ${ }^{* *}$, Lukas Kindl ${ }^{* * *}$, Anja Speyer ${ }^{* * *}$,

\title{
Die Verbesserung der touristischen Wettbewerbsfähigkeit von ländlichen Destinationen
}

\author{
“ janis.dinter@student.vibk.ac.at, Institut für Geographie, Universität Innsbruck \\ *vanessa.faerber@student.uibk.ac.at, Institut für Geographie, Universität Innsbruck \\ *** lukas.kindl@student.vibk.ac.at, Institut für Geographie, Universität Innsbruck \\ ****anja.speyer@student.vibk.ac.at, Institut für Geographie, Universität Innsbruck \\ ${ }^{* * * * *}$ juliane.weickert@student.uibk.ac.at, Institut für Geographie, Universität Innsbruck \\ ***** robert.steiger@uibk.ac.at, Institut für Geographie, Universität Innsbruck \\ ******bruno.abegg@uibk.ac.at, Institut für Geographie, Universität Innsbruck
}

eingereicht am: 25.07.2016, akzeptiert am: 06.10.2016

In vielen ländlichen Regionen spielt der Tourismus eine wichtige Rolle. Diese Rolle (Arbeitsplätze, Einkommen etc.) ist gefährdet, weil immer mehr ländliche Destinationen Mühe bekunden, im globalisierten Tourismusmarkt konkurrenzfähig zu bleiben. Im vorliegenden Artikel wird die touristische Wettbewerbsfähigkeit von zwei ländlichen Destinationen in Niederösterreich mit Hilfe der Importance-Performance-Analyse untersucht. Ziel ist es, die Stärken und Schwächen der beiden Regionen in Bezug auf ihre Wettbewerbsfähigkeit herauszuarbeiten, aber auch die Vor- und Nachteile der angewandten Methodik zu diskutieren.

Keywords: ländlicher Tourismus, Wettbewerbsfähigkeit, Importance-Performance-Analyse, Niederösterreich

\section{Improving the tourism competitiveness of rural destinations}

Tourism plays a major role in many rural areas, creating jobs and income. However, an increasing number of rural destinations are struggling to compete in a globalized tourism market place. In this paper the tourism competitiveness of two rural destinations in Lower Austria is assessed using importance-performance analysis (IPA). The aim is a) to reveal the destinations' strengths and weaknesses, and b) to discuss the suitability of IPA for assessing tourism competitiveness. Results indicate that IPA is a good tool to assess the current situation but less suitable for formulating recommendations to boost competitiveness.

Keywords: rural tourism, competitiveness, importance-performance analysis, Lower Austria

\section{$1 \quad$ Hintergrund und Ziel des Beitrags}

Tourismus ist ein wichtiges Element im Portfolio von Städten, Regionen und Nationen. Erfolg vorausgesetzt, kann der Tourismus maßgeblich zu Wohlstand und Lebensqualität beitragen (Crouch \& Ritchie 1999). In Österreich ist die Tourismuswirtschaft für ca. 7,7\% des nationalen BIP verantwortlich (Statistik Austria 2016) und spielt somit eine wesentliche Rolle für den Wohlstand des Landes. Touristischer Erfolg ist eng mit touristischer Wettbewerbsfähigkeit verknüpft. Lediglich eine touristische Grundinfra- struktur zur Verfügung zu stellen, reicht schon lange nicht mehr aus. Die multioptionalen Gäste von heute setzen ein vielfältiges, qualitativ hochwertiges und gleichzeitig kostengünstiges Angebot voraus. Nur: attraktiv und wettbewerbsfähig zu bleiben, wird aufgrund des wachsenden touristischen Angebots auf dem Weltmarkt immer schwieriger.

In ländlichen Regionen, abseits der etablierten Tourismuszentren, wo dem Tourismus wegen fehlender Alternativen zur Einkommensgenerierung eine noch größere Bedeutung zukommen kann, ist der Wettbewerbsdruck besonders ausgeprägt. Folgende 
Faktoren erschweren die erfolgreiche Positionierung auf dem Markt (vgl. z. B. Rein \& Schuler 2012; Steiger \& Abegg 2015; Zellmann \& Mayrhofer 2015):

- Veraltete Tourismusinfrastruktur

- Veränderung der naturräumlichen Rahmenbedingungen u. a. durch die Folgen des Klimawandels

- Kürzung von Subventionen (Begrenzung der Fördergelder für den Tourismus)

- Demographischer Wandel (Abwanderung, Überalterung) etc.

Diese Herausforderungen sind im südlichen Mostviertel und in den Wiener Alpen, zwei ländlichen Tourismusdestinationen in Niederösterreich, bestens bekannt. Im vorliegenden Artikel geht es daher um die touristische Wettbewerbsfähigkeit dieser beiden Regionen, konkret:

Wie schätzen lokale Stakeholder/innen aus dem Mostviertel und den Wiener Alpen die Wettbewerbsfähigkeit ihrer Destination im Vergleich zur direkten Konkurrenz ein?

Diese Frage soll mit Hilfe einer Importance-Performance-Analyse beantwortet werden. Ziel ist es, die Stärken und Schwächen der beiden Regionen in Bezug auf ihre Wettbewerbsfähigkeit herauszuarbeiten, aber auch die Vor- und Nachteile der angewandten Methodik zu diskutieren.

\section{Niederösterreichische Bergerlebniszent- ren im Mostviertel und den Wiener Alpen}

Bei den Untersuchungsgebieten (Abb. 1), dem südlichen Mostviertel (MV) und den Wiener Alpen (WA), handelt es sich um zwei Tourismusregionen, deren räumliche Ausbreitung noch nicht endgültig fixiert zu sein scheint. Erst im Januar 2016 wurde etwa Wiener Neustadt in das Tourismuskonstrukt Wiener Alpen aufgenommen (Wiener Alpen in Niederösterreich Tourismus GmbH 2015). Die Regionen werden demnach nicht nur durch ihre geographische Ausbreitung definiert und begrenzt, sondern sind vor allem politische bzw. tourismuswirtschaftliche Konstruktionen, welche die bestehenden Grenzen nicht als absolut sehen. Mögliche räumliche Expansionen der Tourismusregionen oder Grenzadaptierungen sind daher auch in Zukunft nicht auszuschließen. Auf die derzeit neun Bergerlebniszentren (BEZ) (vier im südlichen Mostviertel, fünf in den Wiener Alpen) soll sich der touristische Fokus in der Region richten. Diesen BEZ wird von Seiten der Tourismusorganisationen und der Landespolitik am meisten touristisches Potenzial zugesprochen. Das „Bergerlebnis“ ist eine von vier Stoßrichtungen der niederösterreichischen Tourismusstrategie. Durch aktive Standort- und Produktentwicklung soll einerseits die touristische Wettbewerbsfähigkeit des teilweise peripher gelegenen niederösterreichischen Südens gesteigert werden, andererseits sollen positive Impulse für die gesamte Wirtschaft der Region gesetzt werden (NÖ-BBG 2015).

\subsection{Wiener Alpen}

Die Wiener Alpen sind eine österreichische Tourismusregion und können dem niederösterreichischen Industrieviertel zugeordnet werden. Sie bestehen aus urbanem Raum (Wiener Neustadt), hügeligen Landschaften (z. B. Buckelige Welt) sowie den Berglandschaften der nördlichen Ostalpen (etwa die RaxSchneeberg-Gruppe).

Der Begriff der Wiener Alpen leitet sich einerseits davon ab, dass es sich bei den Höhenzügen der Region um die (nordöstlichen) Ausläufer der Ostalpen handelt und andererseits, dass diese die nächstgelegenen Gebirge der Wiener Region sind (abgesehen von den erheblich niedrigeren Kahlenberg, Leopoldsberg oder Bisamberg, die sich in bzw. in unmittelbarer Nähe der Bundeshauptstadt befinden). Aufgrund der geographischen Nähe werden Rax, Schneeberg, Gutensteiner Alpen und Hohe Wand in der Literatur, etwa Wanderführern, vielfach als „Wiener Hausberge“ bezeichnet.

Diese geographische Nähe zu Wien (ca. 100 Kilometer auf den Semmering) führte dazu, dass die Region seit dem 19. Jahrhundert als Ziel der vornehmen Gesellschaft für ihre Sommerfrische fungierte. Orte wie Semmering oder Reichenau an der Rax entwickelten sich zu blühenden Tourismusorten mit prosperierender Wirtschaft. Neben den zwei genannten zentralen Tourismusorten gibt es drei weitere Bergerlebniszentren in den Wiener Alpen: Puchberg am Schneeberg, St. Corona am Wechsel und Mönichkirchen, die derzeit primär auf den Wintertourismus bauen, aber laut Leitstrategie einen Ganzjahrestourismus anstreben sollen (NÖ-BGG 2015). Mit 1. Januar 2016 wurde auch die größte Stadt der Region, Wiener Neustadt, mit ihren etwa 40000 Einwohner/innen in die Tourismusregion Wiener Alpen aufgenommen (Wiener Alpen in Niederösterreich Tourismus GmbH 2015). Damit stellen sich die Wiener Alpen nicht nur landschaftlich, sondern auch strukturell als äußerst heterogene Tourismusregion dar, die ein breites Spektrum an Aktivitäten für Tourist/innen bieten will.

Allerdings hat die Region mit einigen strukturellen Problemen zu kämpfen (etwa Arbeitsplatzmangel, v. a. außerhalb der Tourismusbranche, und touristischer Konkurrenz aus dem In- und Ausland). Eine weitere Herausforderung stellen die Auswirkungen 
des Klimawandels dar. Die vergleichsweise tief gelegenen Skigebiete kämpfen bereits heute mit unsicheren Schneeverhältnissen - im Zuge des Klimawandels wird die Schneesicherheit weiter schwinden (vgl. Steiger \& Abegg 2015).

In den Wiener Alpen leben etwa 200000 Menschen (Bezirke Neunkirchen, Wiener Neustadt-Land sowie Statutarstadt Wiener Neustadt), davon entfallen rund 7000 auf die fünf Bergerlebniszentren der Region (Amt der NÖ Landesregierung 2015). Die Bevölkerungsentwicklung in den Bezirken stagniert (negative Geburtenbilanz, allerdings positive Wanderungsbilanz), und Abwanderungstendenzen von ruralen in (semi-)urbane Gebiete sind erkennbar: Während urbane Zentren, wie Wiener Neustadt, kontinuierlich wachsen (v.a. durch Zuzug), kämpfen kleinere Gemeinden in der Regel mit Überalterung und Wegzug (Amt der NÖ Landesregierung 2015).

Auch touristisch stagnieren die Bergerlebniszentren der Wiener Alpen. So lag das Mittel der Tourismusjahre 2013-2015 (275981) leicht unter dem Wert von 2003-2005 (280501). Die Aufenthaltsdauer blieb unverändert bei 3,0 Tagen und ist damit auch deutlich unter dem österreichischen Durchschnitt (3,4 Tage).

\subsection{Mostviertel}

Das Mostviertel befindet sich im Südwesten Niederösterreichs, wird im Norden von der Donau begrenzt und im Westen und Süden von den jeweiligen Landesgrenzen zu Oberösterreich und der Steiermark. Im Osten grenzt das Mostviertel an den Wienerwald und die Tourismusregion „Wiener Alpen“.

Seinen Namen verdankt die Region der Mostkultur, der seit Jahrhunderten gepflegten Herstellung von vergorenem Apfel- und Birnensaft. Diese kulturelle Besonderheit wird auch touristisch genutzt, so wurde - vor allem für den Fremdenverkehr konzipiert eine ca. 200 Kilometer lange Moststraße im hügeligen Westen der Region eingerichtet. Vierkanthöfe und Mostheurige laden zum Verweilen ein (Mostviertel Tourismus GmbH 2016a).

Einen anderen Schwerpunkt setzt die alpine Urlaubsregion im Süden des Mostviertels. Aufgrund der gebirgigen Landschaft steht hier das Bergerlebnis im Vordergrund. Das südliche Mostviertel ist von den Ausläufern der Nördlichen Kalkalpen, dem Ötschergebiet, geprägt und umfasst die vier Bergerlebniszentren Göstling an der Ybbs, Lackenhof am Ötscher (Gemeinde Gaming), Mitterbach am Erlaufsee und Annaberg (Mostviertel Tourismus $\mathrm{GmbH}$ 2016b).

Im südlichen Mostviertel wird ebenfalls stark auf den Wintertourismus gesetzt, und ähnlich wie in den

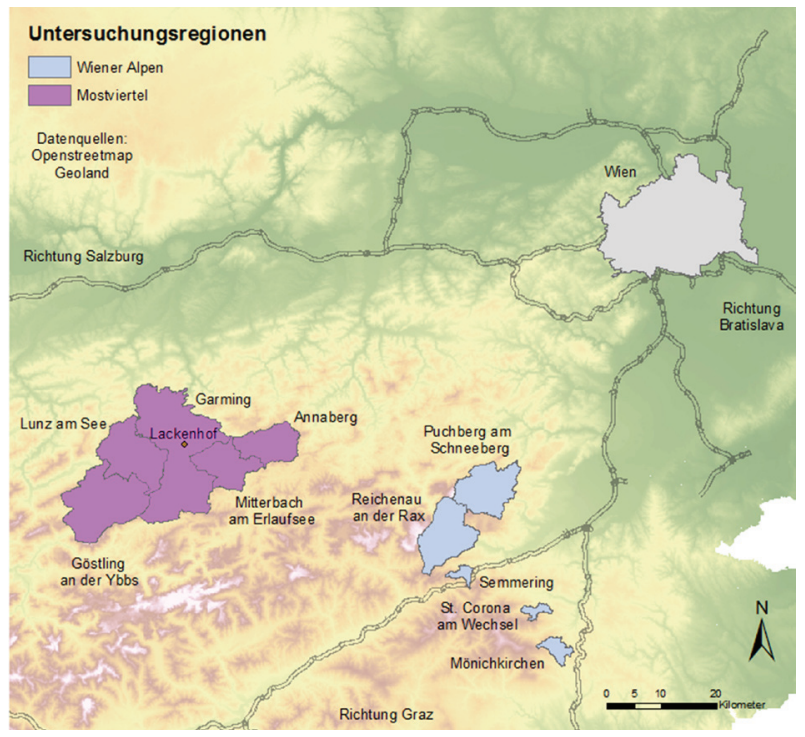

Abb. 1: Übersichtskarte südliches Niederösterreich; eigene Darstellung

Wiener Alpen bekunden auch die Bergerlebniszentren des Mostviertels immer mehr Mühe, mit den höher gelegenen und größeren Wintersportregionen im Westen Österreichs mithalten zu können. Dies spiegelt sich auch in den touristischen Kennzahlen wieder: so mussten die Bergerlebniszentren im Mostviertel einen starken Rückgang von 326978 Übernachtungen im Zeitraum 2003-2005 auf 247395 Übernachtungen (2013-2015) hinnehmen (-24\%). Auch die Aufenthaltsdauer nahm ab - von 3,65 auf 3,21 Tage.

Da der Erhalt der bestehenden Infrastruktur von einem gewissen volkswirtschaftlichen Interesse ist, wurde und wird den kleineren Skigebieten der Region vergleichsweise viel Aufmerksamkeit geschenkt. In Anbetracht der zu erwartenden klimatischen Veränderungen und der damit verbundenen negativen Auswirkungen auf die Schneesicherheit (Steiger \& Abegg 2015), muss die künftige Ausrichtung des Tourismus an manchen Standorten allerdings überdacht bzw. hinterfragt werden.

In den vier Bergerlebniszentren des südlichen Mostviertels leben rund 5000 Einwohner/innen (Amt der NÖ Landesregierung 2015). Die Region ist vor allem durch eine starke Überalterung der Bevölkerung sowie einer gleichzeitig starken Abwanderungsbewegung der jüngeren Bevölkerung geprägt (Amt der NÖ Landesregierung 2016). Gleichbleibende bis sinkende Übernachtungszahlen und eine mangelhafte Verkehrsanbindung tragen außerdem dazu bei, dass die Tourismuszahlen der Region stagnieren und es insgesamt zu keiner spürbar positiven wirtschaftlichen Entwicklung und Dynamik kommt (Wirtschaftskammer Österreich 2015). Die Phänomene der Überalterung und der Wegzug von jungen Leuten sind in vielen ruralen Gebieten Österreichs gegeben, im südlichen 
Mostviertel sind sie allerdings besonders stark ausgeprägt (NÖ-BGG 2015).

\section{Die Importance-Performance-Analyse als Diagnosewerkzeug}

Die Importance-Performance-Analyse (IPA) wurde von John Martilla und John James eingeführt und hat ihren Ursprung im Marketing (Martilla \& James 1977). Sie wird dort zur Evaluierung der Kundenakzeptanz bzw. -zufriedenheit eingesetzt. Dabei bewerten Kundinnen und Kunden bestimmte Faktoren, die für das Produkt bzw. die Dienstleistung relevant sind, nach ihrer Importance (Wichtigkeit) und ihrer Performance (Leistung) (Martilla \& James 1977). Der Status der Faktoren wird durch die Analyse eruiert und grafisch in einer Matrix dargestellt. Die IPA ist ein Diagnosewerkzeug, mit dem man Ergebnisse in konkrete Handlungsempfehlungen „übersetzen“ kann.

Vor einigen Jahren hat die IPA auch Eingang in den Tourismus gefunden (z. B. Deng 2007, Enright $\&$ Newton 2004). In der Tourismusforschung wird die IPA u.a. zur Beurteilung der Wettbewerbsposition eines Produktes, einer Dienstleistung, eines Unternehmens oder einer Destination eingesetzt, um relevante Strategien - für den Wettbewerbsvorteil gegenüber den Konkurrenten - zu formulieren (Azzopardi \& Nash 2013). Wettbewerbsfähigkeit ist eine wesentliche Voraussetzung für den Erfolg einer Tourismusdestination und damit auch ein maßgeblicher Faktor für den ökonomischen Wohlstand und die Lebensqualität in einer Destination (Crouch \& Ritchie 1999).

In der vorliegenden Fallstudie wird davon ausgegangen, dass eine Destination wettbewerbsfähig ist, wenn sie für potentielle Touristinnen und Touristen attraktiv ist und deren Bedürfnissen und Interessen gerecht wird (Enright \& Newton 2004). Man ist in einer wettbewerbsfähigen Destination zudem in der Lage, potenzielle Wettbewerbsvorteile zu identifizieren und für sich zu nutzen bzw. zu verbessern (Azzopardi $\&$ Nash 2013). Die Einwohner/innen profitieren vom Erfolg einer wettbewerbsfähigen Tourismusdestination. Im Sinne eines Trickle-down-Effektes wirkt sich dieser positiv auf die sozialen, ökonomischen und demographischen Aspekte aus.

Das Konzept der "Destination Competitiveness“ nach Crouch und Richtie (1999) trägt durch seinen systematischen Zugang dazu bei, dass Managements befähigt werden, die wettbewerbsrelevanten und einflussgebenden Faktoren einer Tourismusdestination festzulegen. Die Faktoren werden in dem Modell vier verschiedenen Komponenten zugeordnet (Crouch \& Ritchie 1999):
1. Kernressourcen und Attraktoren (Core Resources und Attractors) sind die grundlegenden Elemente der Anziehungskraft einer Destination und machen somit die Schlüsselmotivation für den Besuch einer Destination aus.

2. Unterstützende Faktoren und Ressourcen (Supporting Factors und Resources) bilden die Grundlage, auf welcher die Tourismusindustrie aufbaut.

3. Management der Destination (Destination Management) beeinflusst das Wirken der Komponenten I), II), IV) und V).

4. Qualifizierende und verstärkende Determinanten (Qualifying und Amplifying Determinants) stehen für die situationsbezogenen Bedingungen, die auf die Wettbewerbsfähigkeit Einfluss nehmen. Sie erhöhen oder mindern die Wettbewerbsfähigkeit, indem sie den Einfluss der anderen Komponenten „filtern“.

Crouch (2011) erweiterte den Konzept-Begriff auf die "Tourism Destination Competitiveness" (TDC) und fügte eine fünfte Komponente hinzu:

5. Strategie, Planung und Entwicklung der Destination (Destination Policy, Planning and Development) umfassen die Möglichkeiten bzw. Maßnahmen, die eine Destination umsetzen kann/muss, damit die Ziele erreicht werden.

Die Anzahl und Auswahl der 28 relevanten Importance-Performance-Faktoren wurden vom Forscherteam festgelegt (vgl. Abb. 2). Dabei orientierte man sich maßgeblich an den Publikationen von Crouch \& Ritchie (1999) und Crouch (2011) und traf die entsprechende Auswahl, nachdem man sich mit der jeweiligen Situation der Regionen vertraut gemacht hatte. Mit Hilfe eines Fragebogens bat man 64 Stakeholder/innen (26 im Mostviertel, 38 in den Wiener Alpen) um ihre Einschätzung der eigenen Wettbewerbsfähigkeit. Bei den befragten Personen handelte es sich um ausgewählte Expertinnen und Experten, die in den lokalen Tourismus eingebunden sind (Details dazu siehe Tab. 1). Dazu zählen u. a. Hotel- und Tourismusmanager/innen, Bürgermeister/innen, Gastronom/innen, Obfrauen und Obmänner der örtlichen Tourismusvereine, lokale Einzelhändler/innen etc. Die Befragten mussten in den persönlichen Gesprächen zunächst die Importance der 28 Faktoren auf einer 5er-Likert-Skala - für ihre Tourismusregion einstufen. Danach wurden die Teilnehmer/innen gebeten, die bedeutendsten Konkurrenzdestinationen zu nennen, um anschließend die eigene Performance der 28 Faktoren mit der der Konkurrenz in Relation zu setzen. Als die drei bedeutendsten Konkurrenten sahen die Stakeholder/innen im Mostviertel die Steiermark, die Wiener Alpen sowie Oberösterreich. 
Tab. 1: Befragte Stakeholder nach Bereichen

\begin{tabular}{lrr}
\hline Kategorien & Mostviertel & Wiener Alpen \\
Gastronomie/ & 10 & 19 \\
Beherbergung & & \\
Lokale Politik & 3 & 3 \\
Tourismusvereine & 3 & 3 \\
Touristische Unterneh- & 1 & \\
men (z. B. Skischule) & & 7 \\
Nicht-touristische & 5 & 2 \\
Unternehmen & & $\mathbf{3 8}$ \\
Bergbahnen & 4 & $\mathbf{2 6}$ \\
Gesamt & & \\
\hline
\end{tabular}

In den Wiener Alpen sind die drei wichtigsten Konkurrenten die Steiermark, das südliche Mostviertel und das übrige Niederösterreich. Auffällig in beiden Regionen war, dass einige der Befragten keine Antwort auf die Frage nach den Hauptkonkurrenten wussten: Ihrer Meinung nach ist ihre Region einmalig, unvergleichlich und damit "konkurrenzlos“.

Für die Analyse werden die beiden Untersuchungsregionen separat betrachtet. In einem ersten Schritt werden die Mittelwerte der einzelnen Faktoren berechnet. Die daraus resultierenden Punktepaare (für jeden Faktor einen Importance- und PerformanceMittelwert) werden dann in einem zweiten Schritt in die IPA-Matrix übertragen. Für die Matrix wurde eine datenzentrierte Skalierung gewählt; das heisst, dass der Achsenschnittpunkt den Mittelwerten von Performance (x-Achse) und Importance (y-Achse) entspricht. Aus der Überschneidung der Achsen ergeben sich vier Felder (Quadranten). Jedes dieser Felder schlägt unterschiedliche Handlungsempfehlungen bzw. -strategien vor (Martilla \& James 1977):

Quadrant (A) „Concentrate here“: In diesem Quadranten sind die Faktoren mit einer hohen Importance angeordnet, jedoch kann ihre Performance noch verbessert werden. Die Konzentration von konstruktiven Handlungen kann hier maximale Ergebnisse erzielen und zielt auf eine erfolgreiche Wettbewerbsfähigkeit ab.

Quadrant (B) „Keep up the good work“: In diesem Bereich sind die Faktoren angesiedelt, deren Importance und Performance besonders gut ausgeprägt sind. Der Kurs der Faktoren sollte beibehalten werden, indem man die erfolgreichen Handlungsstrategien aufrechterhält bzw. steigert. Sie sind die Stärken einer Destination und erweisen sich im Wettbewerb als Vorteil (Azzopardi \& Nash 2013).

Quadrant (C) „Low priority“: Faktoren in diesem Quadranten haben sowohl eine niedrige Importance als auch eine niedrige Performance. Sie sind für die Wettbewerbsfähigkeit bzw. den Erfolg einer Destination von untergeordneter Bedeutung (Azzopardi \& Nash 2013). Investitionen in die Optimierung dieser Faktoren drängen sich nicht auf, da ihre Relevanz gering ist.

Quadrant (D) „Possible overkill": Die Performance der Faktoren in diesem Bereich ist höher als ihre Bedeutung. Sie haben einen vergleichsweise geringen Einfluss auf die Wettbewerbsfähigkeit einer Destination (Azzopardi \& Nash 2013). Weitere Investitionen in ihre Performance bzw. die Aufrechterhaltung ihres momentanen Levels „verschwenden“ Ressourcen.

Anhand einer einfachen visuellen Analyse können Entscheidungsträger/innen also die Bereiche identifizieren, in welchen sich die Anstrengungen und Programme (zukünftig) konzentrieren sollen (Martilla \& James 1977).

\section{Die Wettbewerbsfähigkeit der Untersu- chungsregionen}

Die Resultate der IPA sind in Abb. 2 dargestellt, eine tabellarische Auflistung findet sich im Anhang (Tab. 2). Ein Lesebeispiel soll die Interpretation erleichtern: Faktor 25 „Finanzielle Unterstützung durch das Land“ wird im MV (Abb. 2, oben) als überdurchschnittlich wichtig bewertet, und hier weist die Destination auch eine hohe Performance auf, d. h. die Befragten fühlen sich scheinbar durch das Land gut unterstützt. Faktor 24 „gut ausgebildete, regionale Fachkräfte“ ist annähernd so wichtig wie Faktor 25, hier wird die Situation im Mostviertel allerdings als unterdurchschnittlich bewertet.

Faktoren, die sich in den Feldern „A“ und „B“ befinden, weisen eine überdurchschnittliche Wichtigkeit auf. Auffällig ist, dass in beiden Fällen (MV und WA) die Importance der einzelnen Faktoren stets relativ hoch angesiedelt wurde (zwischen 3,7 und 4,9 im MV und 3,9 und 4,8 in den WA, mit einem Mittelwert von 4,4 in beiden Regionen). Die Neigung der Befragten, einzelnen Faktoren eine hohe Wichtigkeit zuzuschreiben, bezeichnet Oh (2001) als „Ceiling Effect". Dennoch sind generelle Tendenzen ablesbar: Faktor 13 „Positive Haltung der Bevölkerung gegenüber dem Tourismus" wird im MV als am Wichtigsten betrachtet, während dies in den WA bei Faktor 1 „Attraktive Kulturlandschaft“ der Fall ist. Beide Regionen stimmen darin überein, dass Faktor 28 „Image“ als sehr wichtig empfunden wird. Die niedrigste Wichtigkeit sehen die Befragten im MV bei den Faktoren 26 „Nähe zu großen Bevölkerungszentren“ und 20 „Starker Binnenmarkt“. In den WA hingegen wird Faktor 12 „Gut ausgebautes ÖV-Netz“ als am wenigsten wichtig bewertet.

Die Performance (also das Leistungsniveau der jeweiligen Region bezüglich der einzelnen Faktoren) 


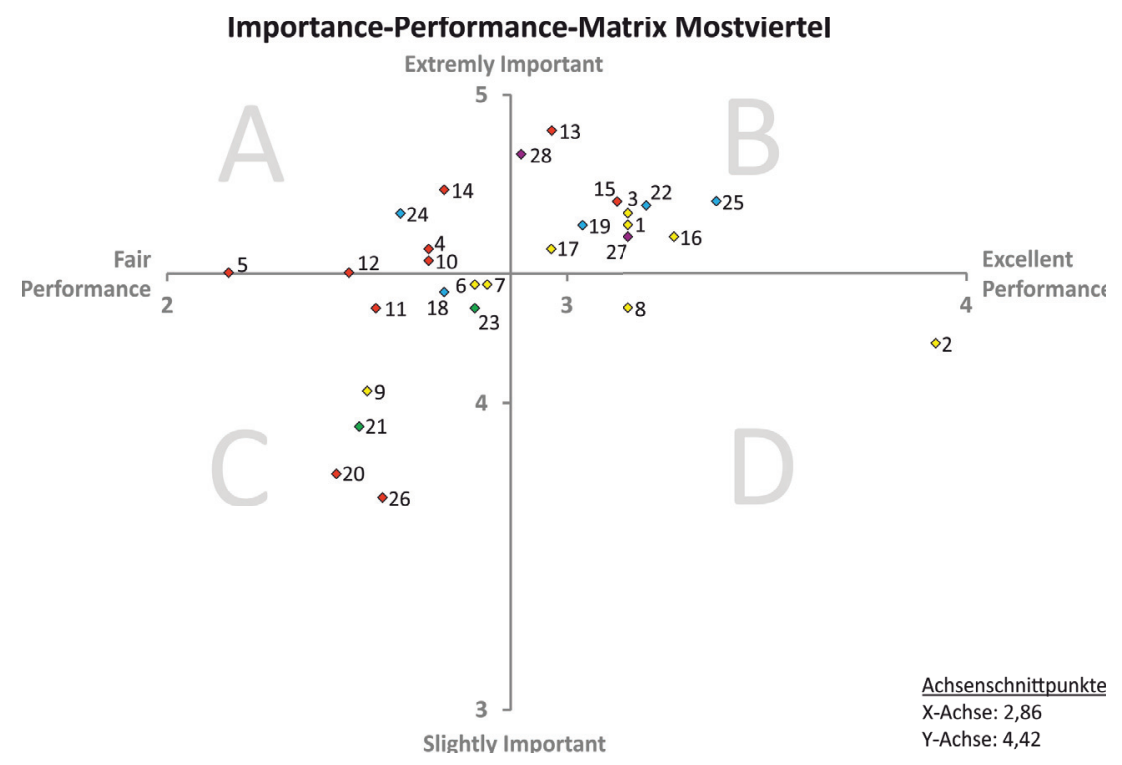

Faktoren

1 Attraktive Kulturlandschaft

2 Unberührte Natur

3 Schneesicherheit

4 Bergbahnen

Vielfältiges Beherbergungsangebot

6 Breite Palette an touristischem Angebot

7 Einzigartige Attraktionen

8 Lebendiges Brauchtum/Reiches Kulturerbe

9 Spezielle Veranstaltungen

10 Gute Erreichbarkeit (PKW)

Gute Erreichbarkeit (ÖV)

Gut ausgebautes ÖV-Netz

Positive Haltung der Bevölkerung ggü. Tourismus

Innovative/tatkräftige UnternehmerInnen im Tourismus

\begin{tabular}{|l|l|}
\hline 15 & Politische Unterstützung \\
\hline 16 & Regionalität \\
\hline 17 & Lebendige, gut funktionierende Dörfer \\
\hline 18 & Schlagkräftige Marketingorganisation \\
\hline 19 & Kooperation zwischen den touristischen AnbieterInnen \\
\hline 20 & Starker Binnenmarkt \\
\hline 21 & Fokussierung auf neue Zielmärkte \\
\hline 22 & Tagesgästlnnen \\
\hline 23 & Klare Positionierung \\
\hline 24 & Gut ausgebildete, regionale Fachkräfte \\
\hline 25 & Finanzielle Unterstützung durch das Land \\
\hline 26 & Nähe zu großen Bevölkerungszentren \\
\hline 27 & Preis-/Leistungsverhältnis \\
\hline 28 & Image \\
\hline
\end{tabular}

\begin{tabular}{|r|l|}
\hline I & Kernressourcen und Attraktoren \\
\hline II & Unterstützenden Faktoren und Ressourcen \\
\hline III & Management der Destination \\
\hline IV & Qualifizierenden und verstärkenden Determinanten \\
\hline V & Strategie, Planung und Entwicklung der Destination \\
\hline
\end{tabular}

Importance-Performance-Matrix Wiener Alpen

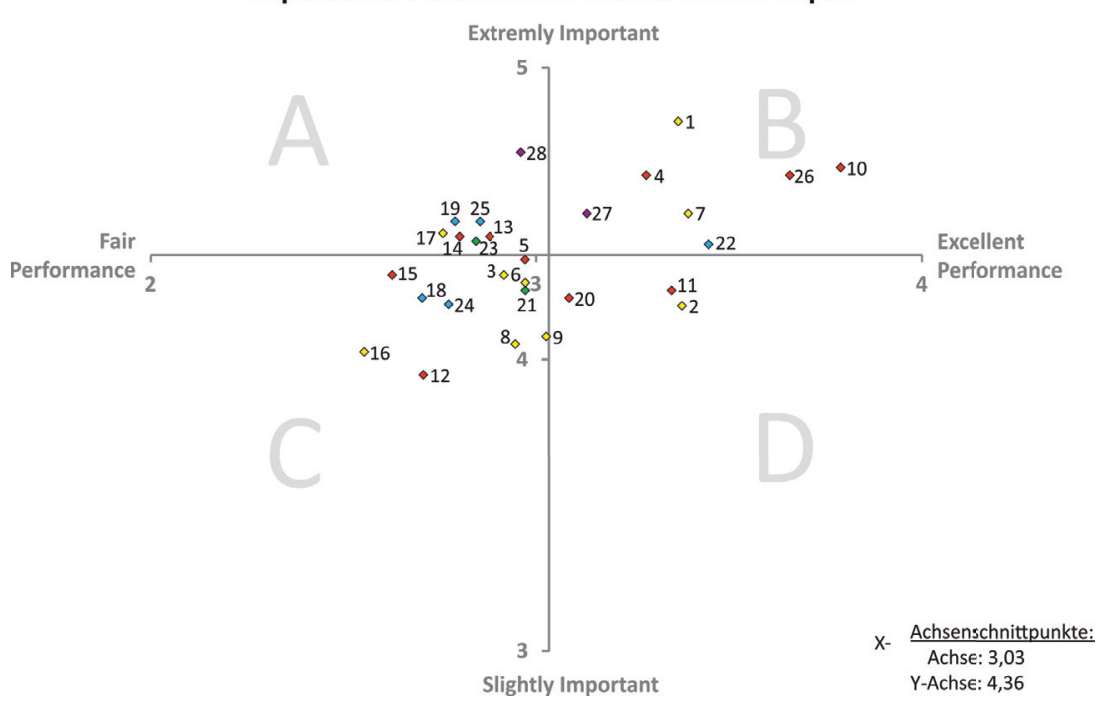

Abb. 2: IP-Matrix für das Mostviertel (oben) und die Wiener Alpen (unten); eigene Darstellung 
vergleicht die eigene Leistung mit der der stärksten Konkurrenten. Faktoren in den Quadranten „B“ und „D“ besitzen ein überdurchschnittliches Leistungsniveau. Der durchschnittliche Wert liegt hier eher im mittleren Bereich der Skala (MV: 2,9 und WA: 3,0). Durch punktuelle Ausreißer ist die Streuung bei der Performance jedoch größer als bei der Importance (zwischen 2,2 und 3,9 im MV und 2,6 und 3,8 in den WA). Folgende Faktoren stechen besonders hervor: Faktor 2 „Unberührte Natur“ ist aus Sicht der Befragten im MV die mit Abstand größte Stärke ihrer Region. Das herausstechende Leistungsniveau dieses Faktors lässt sich in der Importance-Performance-Matrix der Region klar erkennen. Die Befragten in den WA sehen die beiden Faktoren 10 „Gute Erreichbarkeit (PKW)“ und 26 „Nähe zu großen Bevölkerungszentren" als besondere Stärken an. Der letzte Faktor ist durch die Nähe der WA zur Metropolregion Wien gut nachzuvollziehen. Ein schwaches Leistungsniveau sehen die Befragten des MV in Faktor 5 „Vielfältiges Beherbergungsangebot" und jene der WA in Faktor 16 „Regionalität“.

Von besonderer Aussagekraft sind jene Faktoren, bei denen der durchschnittliche Wert der Importance stark von dem der Performance abweicht, d. h. hohes Leistungsniveau bei Faktoren, die tendenziell für weniger wichtig erachtet werden (Quadrant D) und umgekehrt (Quadrant A). In beiden Regionen ist Ersteres bei dem Faktor 2 „Unberührte Natur“ der Fall. In den Wiener Alpen weist zudem Faktor 11 „Gute Erreichbarkeit (ÖV)“ solche Charakteristika auf. Faktoren, die als vergleichsweise wichtig betrachtet werden, die jedoch nur ein niedriges Leistungsniveau erreichen, sind im Mostviertel Faktor 24 „Gut ausgebildete, regionale Fachkräfte“ und Faktor 5 „Vielfältiges Beherbergungsangebot“, in den Wiener Alpen Faktor 19 „Kooperation zwischen den touristischen AnbieterInnen“ und Faktor 17 „Lebendige, gut funktionierende Dörfer" sowie in beiden Regionen Faktor 14 „Innovative/ tatkräftige UnternehmerInnen im Tourismus“. Diese Faktoren verdienen laut Martilla \& James (1977) besondere Beachtung, um die Wettbewerbsfähigkeit im Hinblick auf die Konkurrenz zu erhöhen.

Die Kategorien der TDC aus der Analyse von Crouch \& Richie (1999) und Crouch (2011), die bereits im Methodenkapitel vorgestellt wurden, sind hilfreich bei der Bewertung der vorliegenden Ergebnisse. Die Kategorien bündeln die 28 Faktoren in fünf Gruppen und unterstützen dabei, die folgenden Kernaussagen aus den Matrizen herauszuarbeiten: Sowohl im MV als auch in den WA erhält die Kategorie „Qualifying and Amplifying Determinants" (also: qualifizierende und verstärkende Bestimmungsfaktoren) die durchschnittlich höchsten Importance-Werte. Hierin enthalten sind die Faktoren 27 „Preis-/Leistungsver- hältnis“ und 28 „Image“. Durchschnittlich besonders niedrige Werte erhielten die Faktoren der Kategorie "Destination Policy, Planning and Development" (also: Strategien, Planung und Entwicklung der Tourismusdestination). Hier fällt vor allem das Ergebnis im MV auf, wo sowohl Importance als auch Performance in niedrigen Bereichen liegen. Diese Kategorie umfasst die Faktoren 21 „Fokussierung auf neue Zielmärkte“ und 23 „Klare Positionierung“ und lässt erkennen, dass die Befragten wenig Motivation darin zeigen, die vorherrschenden Verhältnisse zu überarbeiten.

\section{Diskussion der Ergebnisse und Limitati- onen}

Das südliche Mostviertel und die Wiener Alpen stehen vor großen Herausforderungen. Die touristische Entwicklung ist tendenziell rückläufig und alternative Entwicklungspfade sind auch nicht auszumachen. Das Land Niederösterreich hat reagiert und will - nicht zuletzt aus regionalpolitischen Überlegungen heraus - den regionalen Tourismus stützen: nicht via Gießkannenprinzip, sondern unter Fokussierung auf die am besten geeigneten Standorte. Mit gezielten Investitionen, zum Beispiel in die touristische Infrastruktur, sollen das Angebot verbessert und die Wettbewerbsfähigkeit der neun Bergerlebniszentren erhöht werden.

Die IPA bietet eine Möglichkeit, das bestehende Angebot und die Wettbewerbsfähigkeit zu bewerten. Wer die beiden Regionen etwas kennt, wird nicht verwundert sein, dass im MV die „unberührte $\mathrm{Na}$ tur" und in den WA die „gute Erreichbarkeit (PKW)“ und die „Nähe zu großen Bevölkerungszentren“ als Stärken hervorgehoben werden. Weniger ersichtlich zumindest auf den ersten Blick - ist die Platzierung von anderen Faktoren. Hierzu ein Beispiel: Im MV wird Faktor 3 „Schneesicherheit" sowohl bei der Importance als auch der Performance überdurchschnittlich hoch bewertet. Dass der Faktor in Anbetracht der nach wie vor starken Fokussierung auf den Wintertourismus wichtig ist, liegt auf der Hand. Und dass die Schneesicherheit überdurchschnittlich gut sein soll, kann damit erklärt werden, dass sie relativ zu den genannten Konkurrenten gesehen werden muss. Dass also ein vergleichsweise wenig schneesicheres Gebiet mit anderen, ebenfalls wenig (oder noch weniger) schneesicheren Gebieten verglichen wird. Dies weist auf einen weiter oben erwähnten Punkt hin, nämlich dass viele Befragte Mühe hatten, Konkurrenten zu benennen bzw. nur benachbarte Regionen als Konkurrenten benannten. Die beiden Regionen werden als einmalig betrachtet, und man sieht sich nicht einem nationalen bzw. globalen Wettbewerb ausgesetzt. Diese relativ weit verbreitete Sichtweise ist etwas eigenar- 
tig, gilt doch der Tourismus als Paradebeispiel einer globalisierten Industrie. Mit anderen Worten: Auch das MV und die WA stehen im Winter mit den größeren und weitaus besser ausgebauten Wintersportzentren im Westen Österreichs und im Sommer quasi mit der ganzen touristischen Welt in Konkurrenz.

Interessant ist auch Faktor 25 „Finanzielle Unterstützung durch das Land“. Dieser Faktor wird in beiden Regionen als überdurchschnittlich wichtig erachtet. Im MV ist die Performance über-, in den WA unterdurchschnittlich. Letzteres lässt sich vermutlich damit erklären, dass sich einige Leute in den WA benachteiligt fühlen bzw. dass einzelne BEZ mehr Förderung vom Land bekamen als andere. Abgesehen davon drückt die hohe Bewertung dieses Faktors eine nicht $\mathrm{zu}$ vernachlässigende Abhängigkeit von staatlichen Fördermitteln aus. Und wenn man mit den Leuten spricht, kann man sich des Eindrucks nicht erwehren, dass sich über die letzten Jahre auch eine entsprechende Erwartungshaltung entwickelt hat. Dies ist doppelt gefährlich, weil a) Teile des Tourismus offenbar nur mit staatlicher Unterstützung funktionieren, und b) das Land Niederösterreich über eine Reduktion des finanziellen Engagements nachdenkt.

In der Tourismusforschung wird die IPA immer wieder eingesetzt. Die Vorgehensweise ist relativ simpel; Design und Durchführung sind also auch mit beschränkten Ressourcen gut möglich. Mit der IPA können verschiedene qualitative Faktoren, welche in unserem Fall an die Faktoren der Tourism Destination Competitiveness von Crouch (2011) angelehnt sind, quantifiziert und miteinander in Bezug gesetzt bzw. verglichen werden. Als weitere Stärken gelten die übersichtliche Ergebnispräsentation in Form einer Matrix sowie - basierend auf den vier Quadranten dieser Matrix - das vergleichsweise einfache Ableiten von strategischen Handlungsempfehlungen (Azzopardi \& Nash 2013).

Die meisten Vorteile der IPA lassen sich durch unsere Erfahrungen in den beiden Untersuchungsgebieten bestätigen. Dennoch sind einige methodenkritische Bemerkungen - vor allem in Bezug auf die Interpretation - angebracht: Da die Auswahl der Faktoren vorgegeben wurde, sind diese quasi per Definition wichtig, entsprechend hoch sind die Importance-Werte. Die befragten Personen konnten die Liste ergänzen, haben von dieser Möglichkeit aber nicht Gebrauch gemacht, ergo scheint die Vorauswahl akzeptabel. Aber selbst wenn die Faktoren gut gewählt wurden, bedeutet dies nicht, dass alle Faktoren den gleichen Einfluss auf die Wettbewerbsfähigkeit haben. Hinzu kommt, dass die Positionierung der Faktoren in der Matrix eine gewisse „Absolutheit" vorspiegelt. Mal abgesehen davon, dass der Achsenschnittpunkt unterschiedlich definiert werden kann (Azzopardi \&
Nash 2013), stellt sich Frage, wie Faktoren, die auf oder nahe der Achsen liegen, interpretiert werden sollen. Die Bezeichnung der Quadranten mit gängigen Slogans wie Possible overkill oder Keep up the good work suggeriert ferner eine Einfachheit in der Formulierung von Handlungsempfehlungen, die der komplexen Wirklichkeit von unter Wettbewerbsdruck stehenden Tourismusdestinationen nur bedingt gerecht wird.

Es gibt natürlich noch zahlreiche weitere Methoden, die für die Ermittlung der Wettbewerbsfähigkeit im Tourismus herangezogen werden können. Porters (1980) „Fünf Kräfte“ etwa, welche von zahlreichen Autoren angepasst und angewandt wurde, oder die "competitive destination analysis" (CDA) von Pearce (1997). Gemein haben all diese Methoden aber, dass die Bewertung entweder von Touristikerinnen und Touristikern vorgenommen wird, die wie oben erwähnt eine unterschiedliche Sachkenntnis aufweisen können, oder die Bewertung wird von den Wissenschaftlerinnen und Wissenschaftlern selbst, letztlich also subjektiv, vorgenommen.

\section{Fazit}

Ländliche Regionen wie das südliche Mostviertel und die Wiener Alpen müssen um ihre Wettbewerbsfähigkeit in einem globalisierten Tourismusmarkt bangen. Wettbewerbsfähigkeit ist ein komplexer Begriff und Modelle wie dasjenige von Crouch und Ritchie (1999) bzw. Crouch (2011) helfen dabei, diese Vielschichtigkeit greifbar zu machen. Mit der IPA steht außerdem eine Methode zur Verfügung, mit der die verschiedenen Facetten der touristischen Wettbewerbsfähigkeit gemessen werden können. Für die IPA spricht, dass sie ein einfaches und anschauliches Diagnosewerkzeug ist. Mit ihrer Hilfe können verschiedene qualitative Aspekte "quantifiziert" und in einer leicht zu lesenden Matrix dargestellt werden. Die dabei erzielten Resultate geben einen guten Überblick über den relativen Stellenwert der verschiedenen Faktoren; sie decken sich auch mit den aus anderen Quellen (z. B. Lokalaugenschein, Literatur und diverse Gespräche mit Tourismusverantwortlichen des Landes) gewonnenen Erkenntnissen, taugen aber nur beschränkt für die Formulierung von konkreten Handlungsanweisungen. In diesem Sinne ist die oft gerühmte Stärke der Methode - ihre Einfachheit und Übersichtlichkeit Fluch und Segen zugleich: Auf der einen Seite hilft die IPA komplexe Zusammenhänge zu veranschaulichen und fassbar zu machen, auf der anderen Seite verleitet genau diese Vereinfachung zu vorschnellen und simplifizierenden Schlüssen.

Mit der IPA werden zwar die wichtigen Faktoren der Wettbewerbsfähigkeit erfasst, die relative Bedeu- 
tung der einzelnen Faktoren in Bezug auf die GesamtWettbewerbsfähigkeit bleibt aber unberücksichtigt. Hinzu kommt, dass die IPA auch keine Auskunft über die Ursachen der aktuellen Situation macht und sowohl die tourismusinternen als auch die tourismusexternen Rahmenbedingungen außer Acht lässt. Diese müssen jedoch berücksichtigt werden, will man a) das regionale Tourismussystem begreifen und b) den regionalen Gegebenheiten angepasste Strategien formulieren. Mit anderen Worten: Für eine Ist-Analyse der Wettbewerbsfähigkeit ist die IPA gut geeignet; für die Formulierung von konkreten Handlungsanweisungen zur Verbesserung der Wettbewerbsfähigkeit muss sie mit weiterführenden Abklärungen ergänzt werden. Hierbei müssten bspw. die Faktoren gewichtet werden, die Empfehlungen müssen auf die betrachtete Ebene (Einzelunternehmer, Tourismusverband, Land) und die bestehenden Rahmenbedingungen auf diesen Ebenen angepasst werden. Die IPA kann also ein erster Schritt in diese Richtung sein, der Beginn eines Prozesses zur Steigerung der Wettbewerbsfähigkeit.

\section{Dank}

Wir möchten uns bei allen Studierenden der Vertiefungsrichtung „Alpiner Tourismus im Umbruch“ der Geographie Innsbruck für die tatkräftige Unterstützung bedanken. Ein großer Dank geht auch an die befragten Personen im südlichen Mostviertel und in den Wiener Alpen - ohne ihre Auskünfte und Einschätzungen wäre diese Untersuchung nicht möglich gewesen.

\section{Literaturverzeichnis}

Amt der NÖ Landesregierung (2015): Zahlen und Fakten. http://www.noe.gv.at/Land-Zukunft/ZahlenFakten/Land-Bezirke-Gemeinden/NOE_Statistik.html (02.05.2016).

Amt der NÖ Landesregierung (2016): Zahlen und Fakten. http://www.noe.gv.at/Land-Zukunft/Zahlen-Fakten/ Bevoelkerung/Bevoelkerung.html (10.01.2016).

Azzopardi, E. \& R. Nash (2013): A critical evaluation of importance-performance analysis. In: Tourism Management 35, 222-233.

Crouch, G.I. \& J.R.B. Ritchie (1999): Tourism, Competitiveness, and Societal Prosperity. In: Journal of Business Research 44, 137-152.

Crouch, G.I. (2011): Destination Competitiveness: An Analysis of Determinant Attributes. In: Journal of Travel Research 50 (I), 27-45.
Deng, W. (2007): Using a revised importance-performance analysis approach: The case of Taiwanese hot springs tourism. In: Tourism Management 28, 1274-1284.

Enright, M. \& J. Newton (2004): Tourism destination competitiveness: a quantitative approach. In: Tourism Management 25, 777-788.

Martilla, J. \& J. James (1977): Importance-Performance Analysis. In: Journal of Marketing 41 (1), 77-79.

Mostviertel Tourismus GmbH (2016a): Moststraße. http:// www.mostviertel.at/moststrasse (20.06.2016).

Mostviertel Tourismus GmbH (2016b): Schifahren im Mostviertel. http://www.mostviertel.at/ski-snowboard (27.05.2016).

NÖ-BBG - Niederösterreichische Bergbahnen Beteiligungsgesellschaft m.b.H. (2015): Bergerlebnis in Niederösterreich. http://www.noe-bbg.at/bergerlebnisin-niederoesterreich (03.06.2016).

Oh, H. (2001): Revisiting importance-performance analysis. In: Tourism Management 22, 617-627.

Pearce, D.G. (1997): Competitive destination analysis in Southeast Asia. In: Journal of Travel Research 35 (4), 16-24.

Porter, M.E. (1980): Competitive Strategy: Techniques for Analyzing Industries and Competitors. Free Press. New York.

Rein, H. \& A. Schuler (2012): Tourismus im ländlichen Raum. In: Rein, H. \& A. Schuler (Hrsg.): Tourismus im ländlichen Raum. Wiesbaden, 3-10.

Statistik Austria (2016): Das System der Volkswirtschaftlichen Gesamtrechnung http:// www.statistik.at/web_de/statistiken/wirtschaft/ volkswirtschaftliche_gesamtrechnungen/index.html (20.04.2016).

Steiger, R. \& B. Abegg (2015): Klimawandel und Konkurrenzfähigkeit der Skigebiete in den Ostalpen. In: Egger R. \& K. Luger (Hrsg.): Tourismus und mobile Freizeit Lebensformen, Trends, Herausforderungen. Norderstedt, 319-332.

Wiener Alpen in Niederösterreich Tourismus GmbH (2015): Wiener Neustadt bereichert die Wiener Alpen in Niederösterreich Tourismus GmbH. http:// www.wieneralpen.at/wiener-neustadt-bereichert-diewiener-alpen-in-niederoesterreich-tourismus-gmbh (27.05.2016).

Wirtschaftskammer Österreich (2015): Daten für Österreichs Tourismus. https://www.wko.at/Content. Node/Interessenvertretung/ZahlenDatenFakten/Daten_ fuer_Oesterreichs_Tourismus.html (02.05.2016).

Zellmann, P. \& S. Mayrhofer (2015): Die Urlaubsrepublik. Die Zukunft des Tourismus in Österreich. Manz. Wien. 


\section{Anhang}

Tab.2: Mittelwerte und Varianzkoeffizienten der IPA Faktoren

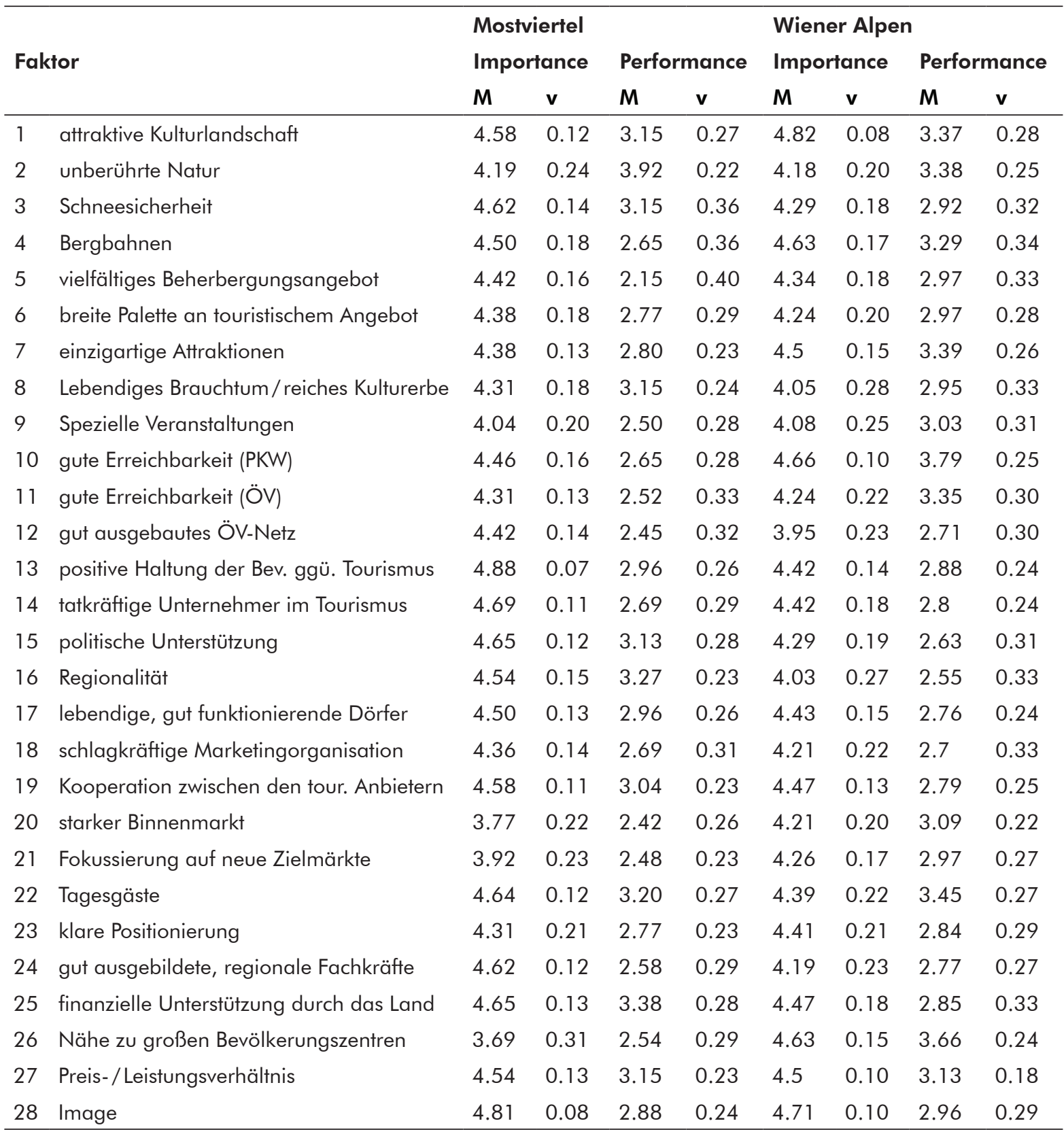

$M=$ arithmetischer Mittelwert $;$ = Varianzkoeffzient 